\title{
CONE VALUED LYAPUNOV FUNCTIONS AND LIPSCHITZ STABILITY OF NONLINEAR SYSTEMS OF DIFFERENTIAL EQUATIONS
}

\section{OLUSOLA AKINYELE}

Crawford Science Hall

Bowie State University

Bowie, MD 20715

USA

(Received October 4, 1994)

ABSTRACT. We introduce a new comparison result which will be an important tool when we apply cone valued Lyapunov like functions. We also introduce new concepts of $\phi_{0}$-uniform Lipschitz stability and $\left(\lambda, \lambda, \phi_{0}\right)$-practical stability and employ our comparison result to carry out stability analysis of nonlinear systems. Our results are also applicable to nonlinear perturbed systems.

KEY WORDS AND PHRASES. Cone valued Lyapunov functions, $\phi_{0}$-uniform Lipschitz stability, practical stability, nonlinear differential equations.

1991 AMS SUBJECT CLASSIFICATION CODES. 34D.

\section{INTRODUCTION.}

The notion of Lipschitz stability in differential equations was introduced by Dannan and Elaydi $[4,5]$. They obtained conditions for the Lipschitz stability of nonlinear systems using the techniques of scalar Lyapunov functions. This concept of stability coincides with uniform stability in linear systems [4] and lies somewhere between uniform stability and both asymptotic stability in variation [2] and uniform stability in variation [3] for nonlinear systems. Moreover, one important feature of Lipschitz stability is that unlike uniform stability the linearised system inherits the property of Lipschitz stability from the original nonlinear system [4].

It is well known that the method of vector Lyapunov functions offers a very flexible and effective mechanism to investigate qualitative properties of nonlinear differential equations $[8,9,10,11,13,14]$. However, in spite of the effectiveness of the method, the limitation is obvious $[6,7,12]$. To circumvent this limitation, it was suggested [7], that employing arbitrary cones rather than the standard cone $\mathbb{R}_{+}^{n}$ which is utilized in the method of vector Lyapunov functions will be beneficial. Above all, it is now well known that employing cone valued Lyapunov functions is beneficial in applications $[1,6,7,12]$.

We shall here introduce a new comparison result which will be an important tool when we apply cone valued Lyapunov like functions. We also introduce new concepts of $\phi_{0}$-uniform Lipschitz stability and $\left(\lambda, \lambda, \phi_{0}\right)$-practical stability and use our comparison result to carry out stability analysis of nonlinear systems. Our results are also applicable to nonlinear perturbed systems. 


\section{PRELIMINARIES.}

We consider the differential systems:

$$
\begin{array}{ll}
y^{\prime}=f(t, y) & y\left(t_{0}\right)=x_{0} \\
x^{\prime}=F(t, x) & x\left(t_{0}\right)=x_{0}
\end{array}
$$

where $f, F: \mathbb{R}_{+} \times \mathbb{R}^{n} \rightarrow \mathbb{R}^{n}$ are assumed continuous.

Let $K \subset \mathbb{R}^{n}$ be a cone, that is, $K$ is closed, convex, with non-empty interior, and satisfies the conditions $K \cap-K=\{0\}$, and $\lambda K \subset K$ for all $\lambda>0$. For any $x, y \in \mathbb{R}^{n}$, we let $x \leq y$ iff $y-x \in K$ and for any functions $u, v: \mathbb{R}_{+} \rightarrow \mathbb{R}^{n}, u \leq v$ iff $u(t) \leq v(t)$ on $\mathbb{R}_{+}$. Let $K^{*}=\left\{\phi \in \mathbb{R}^{n} \mid(\phi, x) \geq 0, \forall x \in K\right\}$, and let $K_{0}^{*}=K^{*} \sim\{0\}$.

DEFINITION 2.1. A function $g: \mathbb{R}^{n} \rightarrow \mathbb{R}^{n}$ is said to be quasi monotone nondecreasing relative to the cone $K$ if $\phi \in K_{0}^{*}$ exists such that $x \leq y$ and $(\phi, y-x)=0$ implies $(\phi, g(y)-g(x)) \geq 0$.

When $K=\mathbb{R}_{+}^{n}$ def. 2.1 says if $x \leq y$ and $y_{\mathfrak{i}}=x_{\mathfrak{i}}$ for some $1 \leq i \leq n$, then $g_{\mathrm{v}}(y)-g_{\mathrm{v}}(x) \geq 0$, which reduces to requiring nonnegative off-diagonal entries of an $n \times n$ matrix $A$ where $g(x)=A x$.

Consider the comparison system:

$$
u^{\prime}=g(t, u) \quad u\left(t_{0}\right)=u_{0}
$$

where $g: \mathbb{R}_{+} \times K \rightarrow \mathbb{R}^{n}$ is assumed continuous. Let $u(t)=u\left(t, t_{0}, u_{0}\right)$ be any solution of the system (2.3). We formulate the following definitions.

DEFINITION 2.2. The differential system (2.3) is said to be $\phi_{0}$-uniformly Lipschitz stable if there exist $M \geq 1, \delta>0$ and $\phi_{0} \in K_{0}^{*}$ such that $\left(\phi_{0}, u\left(t, t_{0}, u_{0}\right)\right) \leq M\left(\phi_{0}, u_{0}\right)$ for $\left(\phi_{0}, u_{0}\right)<\delta$ and $t>t_{0}$.

DEFINITION 2.3. The system (2.3) is $\left(\lambda, \lambda, \phi_{0}\right)$-practically stable if given $0<\lambda<A$, there exists $\phi_{0} \in K_{0}^{*}$ such that $\left(\phi_{0}, u_{0}\right)<\lambda$ implies $\left(\phi_{0}, u\left(t, t_{0}, u_{0}\right)\right)<A, t \geq t_{0}$, where $t_{0} \in \mathbb{R}_{+}$. It is said to be $\left(\lambda, B, T, \phi_{0}\right)$-strongly practically stable if given $0<\lambda<A, B<A$ and $T>0$ there exists $\phi_{0} \in K_{0}^{*}$ such that $u\left(t, t_{0}, u_{0}\right)$ is $\left(\lambda, \lambda, \phi_{0}\right)$-practically stable and $\left(\phi_{0}, u_{0}\right)<\lambda$ implies $\left(\phi_{0}, u\left(t, t_{0}, u_{0}\right)\right)<B$ for $t \geq t_{0}+T$, for some $t_{0} \in \mathbb{R}_{+}$.

Other stability and boundedness definitions based on definitions 2.2 and 2.3 can be formulated.

REMARK 2.4. If $K=\mathbb{R}_{+}^{n}$ and $\phi_{0}=(1,1, \ldots, 1)$, then we have special cases of definitions 2.2 and 2.3. These special cases for $n=1$ reduce to the definition of uniform Lipschitz stability in [5] and practical stability [11].

We now establish a new comparison result.

LEMMA 2.5. Let $g: \mathbb{R}_{+} \times K \rightarrow \mathbb{R}^{n}$ be continuous, and let $g(t, u)$ be quasi monotone nondecreasing in $u$ relative to the cone $K$, for each $t \in \mathbb{R}_{+}$. Let $r(t)$ be the maximal solution of (2.3) relative to $K$ existing on $\left[t_{0}, \infty\right)$ and for $t \geq 0$ and a fixed Dini derivative,

$$
D m(t) \leq_{K} g(t, m(t))
$$

where $m: \mathbb{R}_{+} \rightarrow K$ is continuous. Then $m\left(t_{0}\right) \leq_{K} u_{0}$ implies $m(t) \leq_{K} r(t)$ for $t \geq t_{0}$.

PROOF. Clearly, $D_{-} m(t) \leq_{K} g(t, m(t))$ and so by Theorem 1.5.5 in [13], $m\left(t_{0}\right) \leq_{K} u_{0}$ implies $m(t) \leq_{K} r(t)$ for $t \geq t_{0}$. 
TIIEOREM 2.6. Let $K \subseteq \mathbb{R}^{n}$ be a nonempty, closed, convex cone and assume that:

$\left(\mathrm{H}_{0}\right)$ The solution $y\left(t, t_{0}, x_{0}\right)$ of system $(2.1)$ is unique and continuous with respect to the initial data and is locally Lipschitzian in $x_{0}$.

$\left(\mathrm{H}_{1}\right)$ Let $S(\rho)=\left\{x \in \mathbb{R}^{n} \mid\left\|x-x_{0}\right\|<\rho\right\}, V \in C\left(\mathbb{R}_{+} \times S(\rho), K\right)$ is locally Lipschitzian relative to $K$ and for $t_{0}<s \leq t, x \in \mathbb{R}^{n}, V$ satisfies

$$
D_{-} V(s, y(t, s, x)) \leq_{K} g(t, V(s, y(t, s, x)))
$$

where

$$
D_{-} V(s, y(t, s, x))=\liminf _{h \rightarrow 0^{-}} \frac{1}{h}[V(s+h, y(t, s+h, x+F(s, x)))-V(t, y(t, s, x))]
$$

$\left(\mathrm{H}_{2}\right) \quad g(t, u) \in C\left(\mathbb{R}_{+} \times K, \mathbb{R}^{n}\right)$ and is quasi monotone nondecreasing in $u$ relative to $K$ and the maximal solution $r\left(t, t_{0}, u_{0}\right)$ of $(2.3)$ exists for $t \geq t_{0}$.

Then if $x(t)=x\left(t, t_{0}, x_{0}\right)$ is any solution of $(2.2)$ we have $V\left(t, x\left(t, t_{0}, x_{0}\right)\right) \leq_{K} r\left(t, t_{0}, u_{0}\right), t \geq t_{0}$, provided $V\left(t_{0}, y\left(t, t_{0}, x_{0}\right)\right) \leq_{K} u_{0}$.

PROOF. Let $x(t)$ be any solution of (2.2) and set

$$
m(s)=V(s, y(t, s, x(s)))
$$

where $t_{0} \leq s \leq t$. Thus $m\left(t_{0}\right)=V\left(t_{0}, y\left(t, t_{0}, x_{0}\right)\right)$. So if $V\left(t_{0}, y\left(t, t_{0}, x_{0}\right)\right) \leq_{K} u_{0}$, then $m\left(t_{0}\right) \leq_{K} u_{0}$, and

$$
\begin{aligned}
m(s+h)-m(s)= & V(s+h, y(t, s+h, x(s+h)))-V(s+h, y(t, s+h, x(s)+h F(s, x(s)))) \\
& +V(s+h, y(t, s, x(s)+h F(s, x(s))))-V(s, y(t, s, x(s)))
\end{aligned}
$$

Therefore, if $t_{0} \leq s \leq t$, then

$$
D^{+} m(s) \leq_{K} g(s, V(s, y(t, s, x(s))))=g(s, m(s))
$$

By Lemma 2.5, $V(s, y(t, s, x(s))) \leq_{K} r\left(s, t_{0}, u_{0}\right)$ for $t_{0}<s \leq t$, provided $V\left(t_{0}, y\left(t, t_{0}, x_{0}\right)\right) \leq_{K} u_{0}$. Now $V(t, y(t, t, x(t)))=V\left(t, x\left(t, t_{0}, x_{0}\right)\right)$, so if we set $s=t$, we have $V\left(t, x\left(t, t_{0}, x_{0}\right)\right) \leq_{K} r\left(t, t_{0}, u_{0}\right)$, $t \geq t_{0}$.

REMARK 2.7. (i) $V\left(t_{0}, y\left(t, t_{0}, x_{0}\right)\right)=u_{0}$ implies $V\left(t, x\left(t, t_{0}, x_{0}\right)\right) \leq_{K} r\left(t, t_{0}, V\left(t_{0}, y\left(t, t_{0}, x_{0}\right)\right)\right)$, $t_{0} \leq t<T$ which shows the connection between the solutions of systems (2.1) and (2.2) in terms of the maximal solution of (2.3) relative to the cone $K$.

(ii) If $K=\mathbb{R}_{+}^{n}$, then Theorem 2.6 reduces to Theorem 2.1 in [13] and so our result is an extension of the main comparison theorem in [13] to cone-valued Lyapunov functions.

(iii) Let $P, Q$ be cones in $\mathbb{R}^{n}$ such that $P \subset Q$ and suppose the assumptions of Theorem 2.6 hold with $K=P$, then if $V\left(t_{0}, y\left(t, t_{0}, x_{0}\right)\right) \leq_{P} u_{0}$, we get $V\left(t, x\left(t, t_{0}, x_{0}\right)\right) \leq_{K} r\left(t, t_{0}, V\left(t_{0}, y\left(t, t_{0}, x_{0}\right)\right)\right)$, for $t \geq t_{0}$. If however $Q=\mathbb{R}_{+}^{n}$, we have a component wise estimate.

(iv) The trivial function $f(t, y) \equiv 0$ is admissible in Theorem 2.6. In that case, Theorem 2.6 reduces to Theorem 3.1.3 in [9].

3. APPLICATION TO STABILITY ANALYSIS.

We shall now present results on practical stability and uniform Lipschitz stability of the system (2.2) using our comparison theorem. 
THEOREM 3.1. Assume that $\left(\mathrm{H}_{0}\right)$ of Theorem 2.6 holds.

(I) Let $V \in C\left(\mathbb{R}_{+} \times \mathbb{R}^{n}, K^{\prime}\right)$ and $V(t, x)$ is locally Lipschitzian in $x$ relative to $K$,

(II) $g \in C\left(\mathbb{R}_{+} \times K, \mathbb{R}^{n}\right)$ and for $(t, u) \in \mathbb{R}_{+} \times K, D^{+} V(t, u) \leq_{K} g(t, V(t, u))$, where $g$ is quasi monotone nondecreasing in $u$ relative to $K$ for each $t \in \mathbb{R}_{+}$,

(III) For $(t, x) \in \mathbb{R}_{+} \times S(\rho)$, and $\phi \in K_{0}^{*}, b(\|x\|) \leq(\phi, V(t, x)) \leq a(\|x\|)$, where $a, b \in \mathbb{K}$, the set of all $a \in C\left(\mathbb{R}_{+}, \mathbb{R}_{+}\right)$, such that $a(r)$ is strictly increasing in $r$ and $a(r) \rightarrow \infty$ as $r \rightarrow \infty$.

(IV) $0<\lambda, B<A$ are given with $a(\lambda)<b(A)$ and the unperturbed system $(2.1)$ is $(\lambda, \lambda)$ uniformly practical stable.

Then the $\left(\lambda, B, T, \phi_{0}\right)$-strong practical stability of system (2.3) implies the $(\lambda, B, T)$-strong practical stability of the perturbed system (2.2).

PROOF. Since (2.3) is $\left(\lambda, B, T, \phi_{0}\right)$-strongly practical stable and given $0<\lambda, B<A$, with $a(\lambda)<b(A)$, we can find $\phi_{0} \in K_{0}^{*}$ such that $\left(\phi_{0}, u_{0}\right)<a(\lambda)$ implies $\left(\phi_{0}, u\left(t, t_{0}, u_{0}\right)\right)<b(A)$ for $t \geq t_{0}$ and $\left(\phi_{0}, u_{0}\right)<a(\lambda)$ also implies $\left(\phi_{0}, u\left(t, t_{0}, u_{0}\right)\right)<b(B)$ for $t \geq t_{0}+T$. Now system (2.1) is $(\lambda, \lambda)$-practical stable (see [14] for definition of uniform practical stability), hence $\left\|x_{0}\right\|<\lambda$ implies $\left\|y\left(t, t_{0}, x_{0}\right)\right\|<\lambda$ for $t \geq t_{0}$, for all $t_{0} \in \mathbb{R}_{+}$. With this choice of $\lambda,\left\|x_{0}\right\|<\lambda$, we claim that $\left\|x\left(t, t_{0}, x_{0}\right)\right\|<A$ for $t \geq t_{0}$ where $x\left(t, t_{0}, x_{0}\right)$ is any solution of (2.2). Were this not true, then a solution $x\left(t, t_{0}, x_{0}\right)$ of (2.2) would exist with $\left\|x_{0}\right\|<\lambda$ and $t_{1}>t_{0}$ such that $\left\|x\left(t_{1}, t_{0}, x_{0}\right)\right\|=A$, $\left\|x\left(t, t_{0}, x_{0}\right)\right\|<A$, where $t_{0} \leq t<t_{1}$. Setting $u_{0}=V\left(t_{0}, y\left(t, t_{0}, x_{0}\right)\right)$, Theorem 2.6 implies that $V\left(t, x\left(t, t_{0}, x_{0}\right)\right) \leq_{K} r\left(t, t_{0}, u_{0}\right)$, for $t \geq t_{0}$. Hence, by (III), and the choice of $\phi_{0} \in K_{0}^{*}$, we have

$$
\begin{aligned}
b(A) & \leq\left(\phi_{0}, V\left(t_{1}, x\left(t_{1}, t_{0}, x_{0}\right)\right)\right) \\
& \leq\left(\phi_{0}, r\left(t_{1}, t_{0}, V\left(t_{0}, y\left(t_{1}, t_{0}, x_{0}\right)\right)\right)\right) \\
& \leq\left(\phi_{0}, r\left(t_{1}, t_{0}, a\left(\left\|y\left(t_{1}, t_{0}, x_{0}\right)\right\|\right)\right)\right) \\
& \leq\left(\phi_{0}, r\left(t_{1}, t_{0}, a(\lambda)\right)\right) .
\end{aligned}
$$

Since $\left(\phi_{0}, u_{0}\right)<a(\lambda)$ implies $\left(\phi_{0}, r\left(t_{1}, t_{0}, a(\lambda)\right)\right)<b(A)$ we arrive at a contradiction; hence the claim. Also for all $t \geq t_{0}$, with $\left\|x_{0}\right\|<\lambda$, and since $\left(\phi_{0}, u_{0}\right)<a(\lambda)$ implies $\left(\phi_{0}, r\left(t, t_{0}, u_{0}\right)\right)<b(B)$ for $t>t_{0}+T$,

$$
\begin{aligned}
b\left(\left\|x\left(t, t_{0}, x_{0}\right)\right\|\right) & \leq\left(\phi_{0}, V\left(t, x\left(t, t_{0}, x_{0}\right)\right)\right) \\
& \leq\left(\phi_{0}, r\left(t, t_{0}, V\left(t_{0}, y\left(t, t_{0}, x_{0}\right)\right)\right)\right) \\
& \leq\left(\phi_{0}, r\left(t, t_{0}, a\left(\left\|y\left(t, t_{0}, x_{0}\right)\right\|\right)\right)\right) \\
& \leq\left(\phi_{0}, r\left(t, t_{0}, a(\lambda)\right)\right)<b(B)
\end{aligned}
$$

Therefore $\left\|x\left(t, t_{0}, x_{0}\right)\right\|<B$ for $t>t_{0}+T$, and the proof is complete.

We now give the following result in respect of uniform asymptotic stability of the system (2.2) the proof of which is straightforward.

THEOREM 3.2. Assume that assumption $\left(\mathrm{H}_{0}\right)$ of Theorem 2.6 holds along with (I), (II) and (III) of Theorem 3.1. Let the zero solution of the unperturbed system (2.1) be uniformly stable. Then the $\phi_{0}$-uniform asymptotic stability of system (2.3) implies the uniform asymptotic stability of system (2.2).

REMARK 3.3. We see that the choice of $F(t, x)=f(t, x)+R(t, x)$ and an application of Theorem 3.2 shows that the uniform asymptotic stability of the perturbed system (2) can be 
achicved even if the unperturbed system (2.1) is only uniformly stable. All we need do is require the comparison system to be $\phi_{0}$-uniformly asymptotically stable (see [1] for the definition of $\phi_{0}$ uniform asymptotic stability of the comparison system). A similar remark can be made of the usefulness of Theorem 3.1 in stability analysis of perturbed systems.

THEOREM 3.4. Assume that

(I) $g \in C\left(\mathbb{R}_{+} \times K, \mathbb{R}^{n}\right), g(t, 0)=0$ and $g(t, u)$ is quasi monotone nondecreasing in $u$ relative to $K$,

(II) $V \in C\left(\mathbb{R}_{+} \times S(\rho), \mathbb{R}^{n}\right), V(t, 0)=0$ and $V(t, x)$ is locally Lipschitzian in $x$ relative to $K^{\circ}$ and for $\phi_{0} \in K^{*}, b(\|x\|) \leq(\phi, V(t, x))$ where $b \in \mathbb{K}$ such that $b(\alpha u) \leq u q(\alpha)$ with $q(\alpha) \geq 1, \alpha \geq$ 1 and $D_{-} V(t, x) \leq_{K} g(t, V(t, x))$ for $(t, x) \in \mathbb{R}_{+} \times S(\rho)$.

If the zero solution of (2.3) is $\phi_{0}$-uniformly Lipschitz stable, then the zero solution of (2.2) is uniformly Lipschitz stable.

PROOF. Assume that the zero solution of (2.3) is $\phi_{0}$-uniformly Lipschitz stable, then there exist $L \geq 1, \delta>0$ and $\phi_{0} \in K_{0}^{*}$, such that $\left(\phi_{0}, u\left(t, t_{0}, u_{0}\right)\right) \leq L\left(\phi_{0}, u_{0}\right)$ for $t \geq t_{0}$ and $\left(\phi_{0}, u_{0}\right)<\delta$. Set $f(t, y) \equiv 0$ in (2.1) with $x_{0}$ chosen such that $u_{0}=V\left(t_{0}, x_{0}\right)$, then $y\left(t, t_{0}, x_{0}\right)=x_{0}$ and hypothesis $\left(\mathrm{H}_{0}\right)$ of Theorem 2.6 is trivially verified. Hence $V\left(t, x\left(t, t_{0}, x_{0}\right)\right) \leq_{K} r\left(t, t_{0}, u_{0}\right)$ and

$$
\begin{aligned}
b\left(\left\|x\left(t, t_{0}, x_{0}\right)\right\|\right) & \leq\left(\phi_{0}, V\left(t, x\left(t, t_{0}, x_{0}\right)\right)\right) \leq\left(\phi_{0}, r\left(t, t_{0}, u_{0}\right)\right) \\
& \leq L\left(\phi_{0}, u_{0}\right) \leq L\left\|\phi_{0}\right\| \cdot\left\|u_{0}\right\| \\
& =L\left\|\phi_{0}\right\| \cdot\left\|V\left(t_{0}, x_{0}\right)\right\| \leq L N\left\|\phi_{0}\right\| \cdot\left\|x_{0}\right\|
\end{aligned}
$$

Hence $\left\|x\left(t, t_{0}, x_{0}\right)\right\|<b^{-1}\left(L N\left\|\phi_{0}\right\| \cdot\left\|x_{0}\right\|\right) \leq q\left(L N\left\|\phi_{0}\right\|\right)\left\|x_{0}\right\|=M\left\|x_{0}\right\|$.

REMARK 3.5. (i) If $K=\mathbb{R}_{+}^{n}$ and $\phi_{0}=(1,1,1, \ldots, 1)$, then Theorem 3.4 is the method of vector Lyapunov functions for uniform Lipschitz stability. If $n=1$, we get Theorem 2.1 in [ 5 ]. (ii) In Theorems 3.1, 3.2, and 3.4 we can employ a general measure for the cone valued Lyapunov function instead of the particular measure $(\phi, V(t, x))$ defined by $\phi \in K_{0}^{*}$. The corresponding results demonstrate the flexibility that can be achieved when dealing with cone valued Lyapunov functions, particularly in relation to perturbed nonlinear differential systems.

ACKNOWLEDGEMENT. The author acknowledges with gratitude the financial support from the University of Maryland System through the Wilson H. Elkins Professorship.

\section{REFERENCES}

1. AKPAN, E.P. and AKINYELE, O. On the $\phi_{0}$-stability of comparison differential systems.

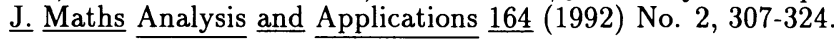

2. BRAUER, F. Perturbations of nonlinear systems of differential equations IV. J. Maths Analysis and Applications 37 (1972) 214-222.

3. BRAUER, F. and STRAUSS, A. Perturbations of nonlinear systems of differential equations III. J. Maths Analysis and Applications $\underline{31}$ (1970) 37-48.

4. DANNAN, F.M. and ELAYDI, S. Lipschitz stability of nonlinear systems of differential equations. J. Maths Analysis and Applications $\underline{113}$ (1986) 562-577.

5. DANNAN, F.M. and ELAYDI, S. Lipschitz stability of nonlinear systems of differential equat-

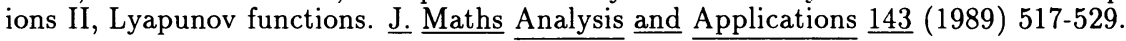


6. KOKSAL, S and LAKSHMIKANTHAM, V. Higher derivatives of Lyapunov functions and cone valued Lyapunov functions. Nonlinear Analysis (to appear).

7. LAKSHMIKANTHAM, V. and LEELA, S. Cone valued Lyapunov functions. Nonlinear Analysis 1 (1977) 215-222.

8. LAKSHMIKANTHAM, V. and LEELA, S. Differential and Integral inequalities Vol. I Academic Press, New York 1969.

9. LAKSHMIKANTHAM, V., LEELA, S., and MARTYNYUK, A. A. Stability Analysis of nonlinear systems. Marcel Dekker Inc 1989.

10. LAKSHMIKANTHAM, V., LEELA, S., and MARTYNYUK, A. A. Practical Stability of nonlinear systems. World Scientific Publications, Singapore, 1990.

11. LAKSHMIKANTHAM, V., MATROSOV, M., and SIVASUNDARAM, S. Vector Lyapunov functions and stability analysis of nonlinear systems. Kluwer Dordrecht, 1991 .

12. LAKSHMIKANTHAM, V. and PAPAGEORGIOU N. S. Cone valued Lyapunov functions and stability theory. Nonlinear Analysis. 22 (1994) no 3, 381-390.

13. RAJALAKSMY S. and SIVASUNDARAM, S. Vector Lyapunov functions and the technique in perturbation theory. J. Maths Analysis and Applications $\underline{164}$ (1992) 560-570.

14. SILJAK, D. D. Large Scale Dynamical systems. North Holland, New York, 1978. 


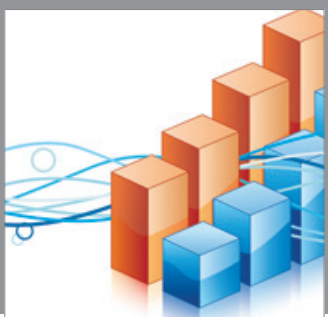

Advances in

Operations Research

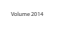

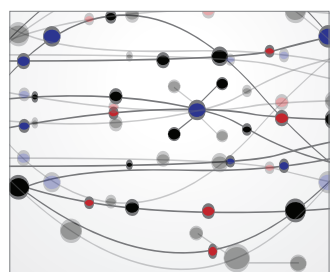

\section{The Scientific} World Journal
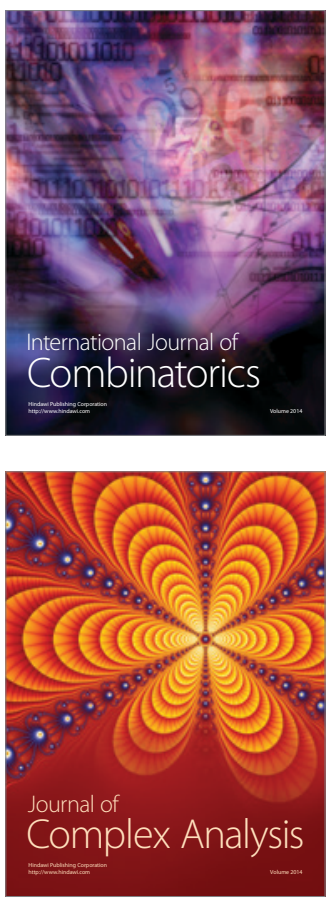

International Journal of

Mathematics and

Mathematical

Sciences
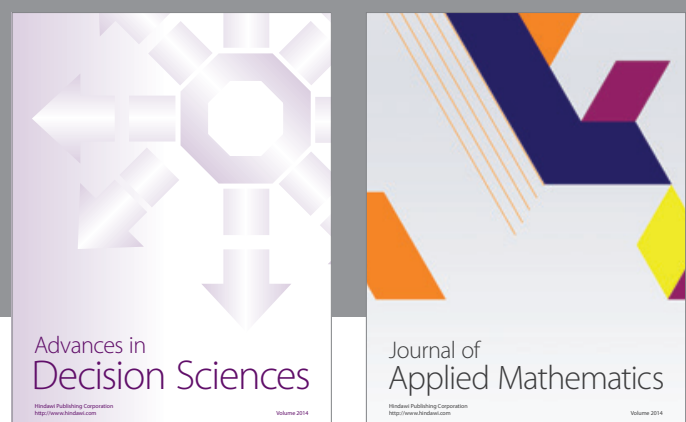

Journal of

Applied Mathematics
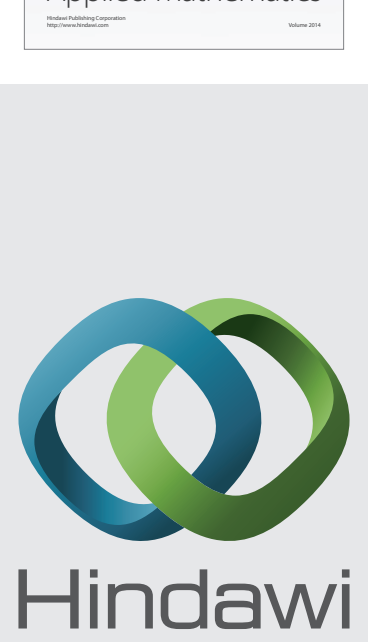

Submit your manuscripts at http://www.hindawi.com
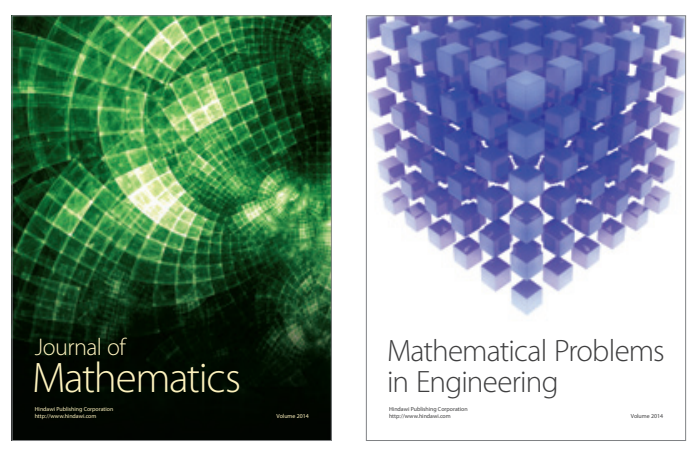

Mathematical Problems in Engineering
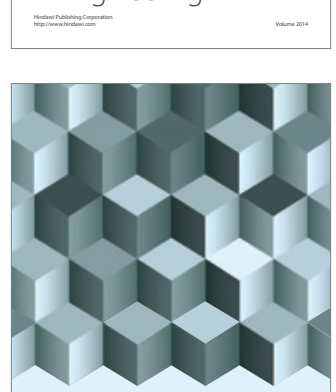

Journal of

Function Spaces
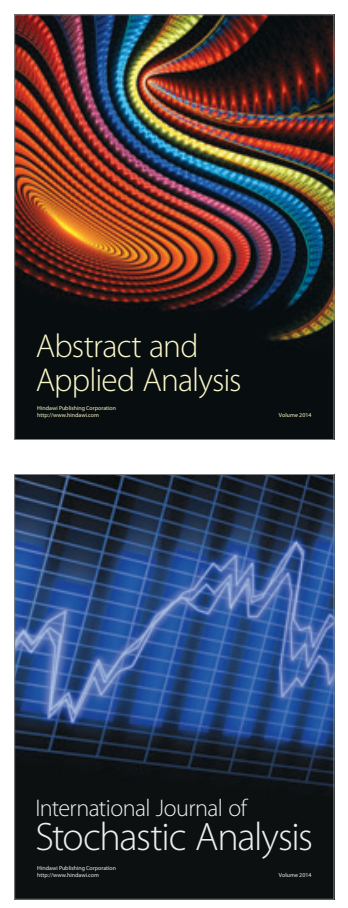

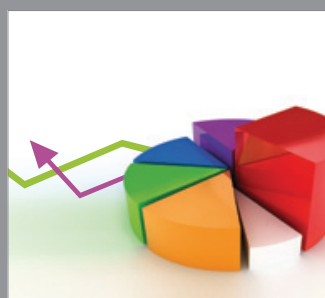

ournal of

Probability and Statistics

Promensencen
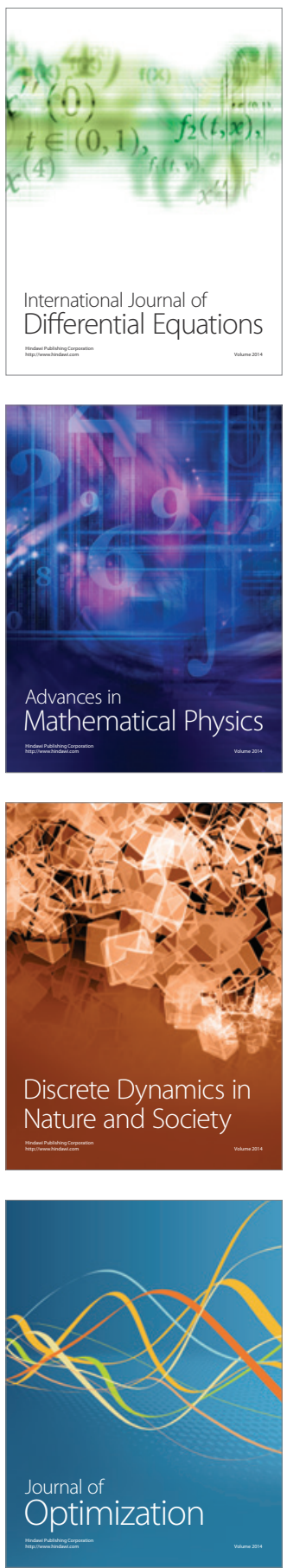